\title{
NIOBIUM THIN FILM PROPERTIES AFFECTED BY DEPOSITION ENERGY DURING VACUUM DEPOSITION
}

\author{
G. $\mathrm{Wu}^{\dagger}$, L. Phillips, R. Sundelin, A-M. Valente \\ Jefferson Lab*, 12000 Jefferson Avenue, Newport News, VA23606, USA
}

\begin{abstract}
In order to understand and improve the superconducting performance of niobium thin films at cryogenic temperatures, an energetic vacuum deposition system has been developed to study deposition energy effects on the properties of niobium thin films on various substrates. Ultra high vacuum avoids the gaseous inclusions in thin films commonly seen in sputtering deposition. A retarding field energy analyzer is used to measure the kinetic energy of niobium ions at the substrate location. A biased substrate holder controls the deposition energy. Transition temperature and residual resistivity ratio (RRR) of the niobium thin films at several deposition energies are obtained together with crystal orientation measurements and atomic force microscope (AFM) inspection, and the results show that there exists a preferred deposition energy around $115 \mathrm{eV}$ (the average deposition energy $64 \mathrm{eV}$ plus the $51 \mathrm{~V}$ bias voltage).
\end{abstract}

\section{INTRODUCTION}

The successful running of LEP2 using a $\mathrm{Nb} / \mathrm{Cu}$ technique demonstrated the significant advantages of niobium thin film technology in particle accelerators [1]. Due to the huge cost savings, a future accelerator for muon/neutrino physics calls for accelerating structures made using thin film technology [2]. While the advantages of a thin film cavity make it very attractive to future accelerator applications, the current magnetron sputtered $\mathrm{Nb} / \mathrm{Cu}$ cavity has much lower $\mathrm{Q}$ value comparing to solid niobium cavities at higher field, [3] which limits the broader adoption for particle accelerators.

Different processes are being tried to improve niobium thin films, including post-deposition laser annealing [4], DC post-magnetron sputtering deposition [5], biased DC magnetron sputtering [6] and vacuum arc deposition [78].

For the aspect of thin film growth, the higher surface adatom mobility [9] and the vacuum condition are believed helpful to get better film quality as required for superconducting material like niobium. For a niobium thin film on a copper substrate, increasing the substrate temperature is not an option to achieve greater surface adatom mobility. Some processes such as ion-assisted deposition, biased magnetron sputtering, ionized magnetron sputtering, vacuum arc deposition and

\footnotetext{
"Work performed under DOE Contract \#DEAC0584ER40150
}

†Electronic mail: genfa@jlab.org energetic cluster deposition, are expected to have higher impact energy during film growth, thus increasing the surface adatom mobility. These techniques either require a working gas or entail risk of microparticle contamination and lack good control of the deposition energy.

To take advantage of the high vacuum condition and the capability of controllable deposition energy, the electron cyclotron resonance (ECR) plasma metal ion source is selected as an energetic deposition system to study the niobium thin film deposition at different deposition energies. To better control the substrate quality, sapphire substrate is used for most of the films. For niobium thin films deposited at different energies, transition temperature and residual resistivity ratio (RRR) are measured. Crystal orientation is checked through Xray diffraction and film surfaces are inspected by an Atomic Force Microscope (AFM).

\section{THE ENERGETIC DEPOSITION SYSTEM}

The metal ion source can be achieved by applying ECR to an evaporated or sputtered metal flux [10-11]. To generate refractory metal ions such as niobium inside a vacuum condition, a thermionic electron gun is used to create the neutral niobium flux, and an ECR chamber is used to convert the neutral niobium atoms to niobium plasma. The energetic niobium ions are extracted to a biased substrate [12]. The niobium ion energy distribution under certain plasma conditions is measured by a retarding field energy analyzer at the substrate location. The result is shown in figure 1 .

The vacuum pressure before the deposition is as low as $9.0 ? 10^{-8}$ torr, changes to 1.0 ? $10^{-6}$ torr during the film deposition in the vacuum chamber and will be significantly lower in the ECR chamber. The majority of the residual gas is hydrogen. The deposition rate averaged $4 \AA$ Ås.

\section{TRANSITION TEMPERATURE MEASURED BY AC INDUCTION}

The transition temperature is obtained through magnetic shielding ability measured by the AC induction method. Care has been taken to minimize the edge effect and the driven signal is at low voltage magnitude as shown in figure 2 (2 volts over 100 ? current limiting resistor).

The transition temperature for films deposited at different bias voltages are shown in figure 3. The transition width result is shown in figure 4. 


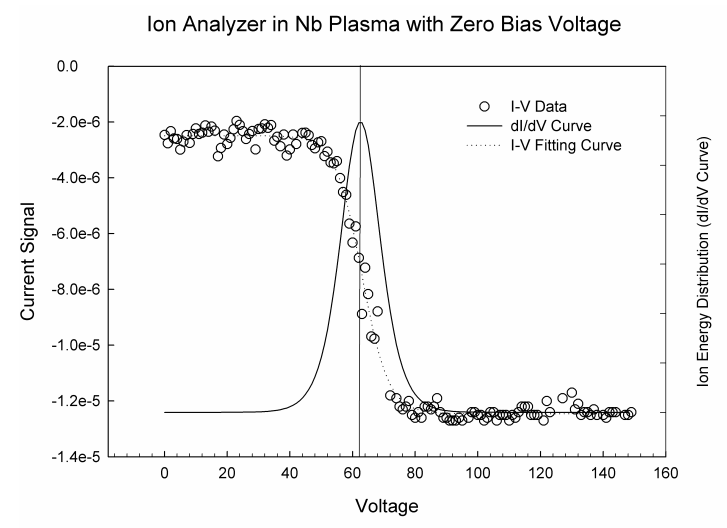

Fig. 1 The ion energy analyzer measurement result for niobium ions at the substrate without bias voltage

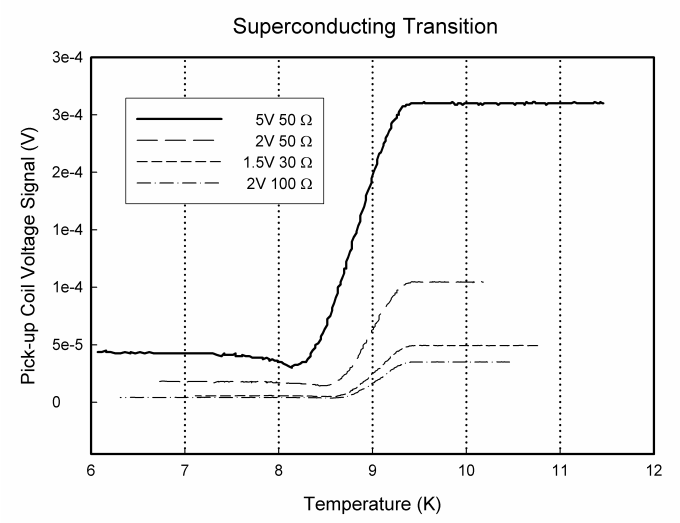

Fig. $2 T_{c}$ curve measured by an induction coil set with different driving signal voltage levels.

\section{$T_{c}$ vs. Bias Voltage}

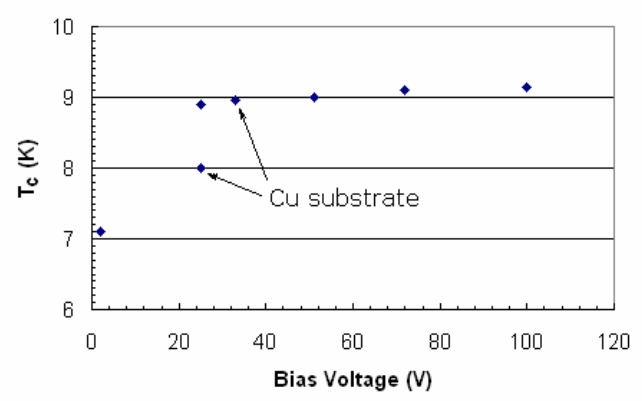

Fig. 3 Bias voltage effect on transition temperature, measured by an inductive method. Substrate is A-cut sapphire unless noted otherwise.

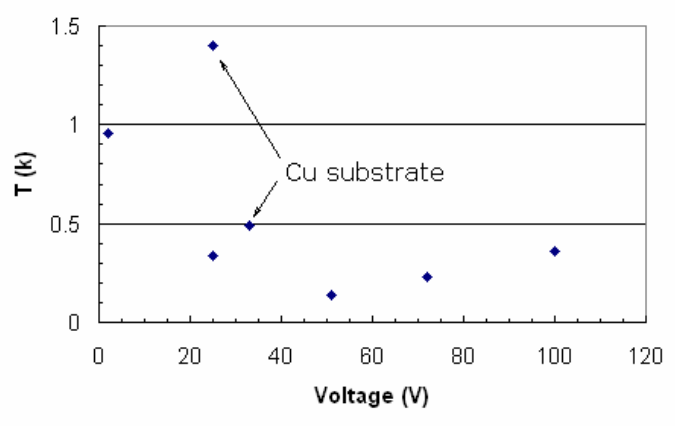

Fig. 4 Bias voltage effect on transition width. Substrate is A-cut sapphire unless noted otherwise.

\section{RRR}

The RRR value has been measured with a four-point resistive method and is defined as the ratio of resistivity at 300 Kelvin to the resistivity at 10 Kelvin. Table 1 shows the RRR result together with the film thickness. The lowered RRR values are likely caused by the increased electron resistance by electron boundary scattering in those very thin films [13].

Table 1: RRR and thickness for different films

\begin{tabular}{|c|c|c|}
\hline $\begin{array}{c}\text { Film batch started with } \\
\text { bias voltage number }\end{array}$ & RRR & Thickness (nm) \\
\hline 02-SA & 2.31 & 68.2 \\
\hline 25-SA & 6.8 & 126 \\
\hline 51-SA & 2 & 74.1 \\
\hline 51-SA & 50.2 & 235 \\
\hline 72-SA & 26.7 & 181 \\
\hline 100-SA & 4 & 79 \\
\hline
\end{tabular}

\section{X-RAY DIFFRACTION AND AFM SURFACE INSPECTION}

$\mathrm{X}$-ray diffraction has been used on the films. The full width of half magnitude (FWHM) value of the diffraction signal for the Nb-110 crystal plane is shown in table 2 .

Table 2. Crystal orientation spread by X-ray diffraction

\begin{tabular}{|c|c|}
\hline $\begin{array}{c}\text { Film batch started with } \\
\text { bias voltage number }\end{array}$ & $\begin{array}{c}\text { Angle FWHM value of } \\
\text { X-ray diffraction signal }\end{array}$ \\
\hline 02-SA & $10-15$ \\
\hline $25-$ SA & $3-4$ \\
\hline $51-$ SA & $<1$ \\
\hline $72-$ SA & $5-6$ \\
\hline $100-$ SA & 5 \\
\hline
\end{tabular}


The film deposited at negative $51 \mathrm{~V}$ substrate bias was checked by an AFM. The surface morphology shows unusually long narrow grains compared with the round dome-shaped grains seen in films at lower substrate bias voltage.

\section{CONCLUSION}

From the results shown in this report, it is concluded that a reasonably high RRR niobium thin film with excellent superconducting transition temperature and width can be achieved through controlled energy deposition in high vacuum. The RRR appears to be more closely related to the film thickness than to the deposition energy. Deposition energy around $115 \mathrm{eV}$ is believed to be the preferred value based on the transition width, the crystal orientation spread, and the AFM results. The AFM surface inspection shows that the surface morphology is affected by the ion impact energy. The X-ray diffraction analysis shows the niobium thin film has a preferred crystal orientation when deposited on sapphire.

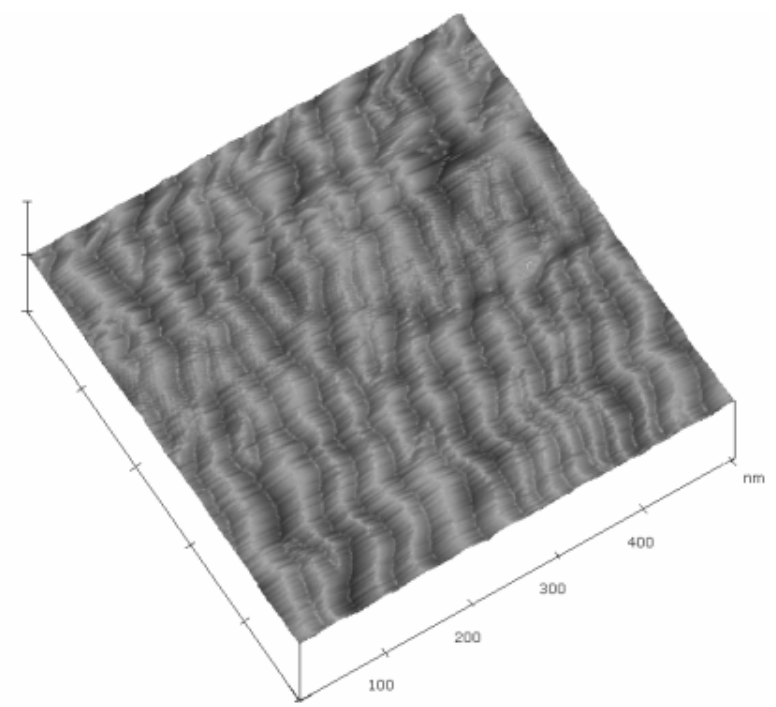

Fig. 5 Long continuous grains of niobium film on sapphire deposited at negative $51 \mathrm{~V}$ substrate bias.

\section{ACKNOWLEDGEMENT}

The authors are very grateful for technical support from Bret Lewis, Sam Morgan, Peter Kushnick, John Musson and many other Jefferson Lab staff members and also Brandt Robertson from Old Dominion University. This work was also helped by L. Hand and made use of the Cornell Center for Materials Research Shared Experimental Facilities, supported through the National Science Foundation Research Science and Engineering Centers program (DMR-9632275).

\section{REFERENCES}

[1]P. Brown, et. al., 9th Workshop on RF Superconductivity, Santa Fe, (1999), edited by B. Rusnak,
Los Alamos National Laboratory, Los Alamos, 1, (1999).

[2]H. Padamsee, 2001 PARTICLE ACCELERATOR CONFERENCE, Chicago, Illinois U.S.A., (2001), edited by P. Lucas, S. Webber, Institute of Electrical and Electronics Engineers, Inc., Piscataway, NJ 08855 , (2001).

[3]V. Arbet-Engels, et al., Nucl. Instrum. Methods Phys. Res. A 463, 1-8 (2001).

[4]E. Radicioni, et al., Nucl. Instrum. Methods Phys. Res. A 365, 28-35 (1995).

[5]V. Palmieri, et al., Proc. of 7th workshop on RF superconductivity, Gif-sur-Yvette, 485, (1995), edited by B. Bonin.

[6]K. Zhao, et al., 9th Workshop on RF Superconductivity, Santa Fe, (1999), edited by B. Rusnak Los Alamos National Laboratory, Los Alamos, 70, (1999).

[7]Y. Igarashi and M. Kanayama, J. Appl. Phys. 57(3), 849-854 (1985).

[8]R. Russo, et al., 10th Workshop on RF Superconductivity, Tsukuba, Japan, (2001), edited by S. Noguchi KEK, Tsukuba, Japan, (2002).

[9]J. A. Thornton, J. Vac. Sci. Technol. 11, 666 (1974).

[10]W. M. Holber, et al., J. Vac. Sci. Technol. A 11, 2903 (1993).

[11]S. M. Rossnagel, et al., J. Vac. Sci. Technol. B 12, 449 (1994).

[12]G. Wu, et al., 10th Workshop on RF Superconductivity, Tsukuba, Japan, (2001), edited by S. Noguchi KEK, Tsukuba, Japan, (2002).

[13]H. M. Rosenburg, Low Temperature Solid State Physics (Oxford University Press, 1963) 